\title{
Topological Nature of Anomalous Hall Effect in Ferromagnets
}

\author{
Masaru OnODA ${ }^{\dagger}$, and Naoto NagaOsA ${ }^{\dagger, *}$, f $^{\prime}$ \\ ${ }^{\dagger}$ Correlated Electron Research Center (CERC), \\ National Institute of Advanced Industrial Science and Technology (AIST), \\ Tsukuba Central 4, Tsukuba 305-8562 \\ *Department of Applied Physics, University of Tokyo, \\ Bunkyo-ku, Tokyo 113-8656
}

\begin{abstract}
The anomalous Hall effect in two-dimensional ferromagnets is discussed to be the physical realization of the parity anomaly in $(2+1) \mathrm{D}$, and the band crossing points behave as the topological singularity in the Brillouin zone. This appears as the sharp peaks and the sign changes of the transverse conductance $\sigma_{x y}$ as a function of the Fermi energy and/or the magnetization. The relevance to the experiments including the three dimensional systems is also discussed.
\end{abstract}

\footnotetext{
${ }^{1}$ E-mail: m.onoda@aist.go.jp

${ }^{2}$ E-mail: nagaosa@appi.t.u-tokyo.ac.jp
} 
Anomalous Hall effect (AHE) in ferromagnets is a phenomenon that there appears the contribution to the transverse resistivity $\rho_{H}$ proportional to the magnetization $M$ in addition to the usual Hall effect [1]:

$$
\rho_{H}=R_{0} B+4 \pi R_{s} M
$$

where $B$ is the magnetic field, $R_{0}$ is the ordinary Hall constant, and $R_{s}$ is the anomalous Hall coefficient. It has been recognized that this AHE comes from the spin-orbit interaction $\lambda$ because some connection between the spin and orbital motion is needed [2, 3, 4, 5, 6]. Karplus and Luttinger (KL) [2] was the first to study theoretically the AHE based on the band theory, and recognized that this effect is due to the inter-band matrix element of the current operators. An important point is that the eigenstates of the Hamiltonian are still the Bloch waves $|n \vec{k}\rangle$ (n: band index, $\vec{k}$ : crystal momentum in the 1st Brillouin zone) even when the spin-orbit interaction is present, namely the translational symmetry remains. Therefore $\sigma_{x y}(\omega)$ for noninteracting electrons is given by [7]

$$
\begin{aligned}
\sigma_{x y}(\omega)=i \sum_{n \neq m} \sum_{\vec{k}} \frac{f\left(\varepsilon_{m}(\vec{k})\right)-f\left(\varepsilon_{n}(\vec{k})\right)}{\varepsilon_{n}(\vec{k})-\varepsilon_{m}(\vec{k})} \\
\times \frac{\left\langle n \vec{k}\left|J_{y}\right| m \vec{k}\right\rangle\left\langle m \vec{k}\left|J_{x}\right| n \vec{k}\right\rangle}{\omega+i \eta+\varepsilon_{m}(\vec{k})-\varepsilon_{n}(\vec{k})}
\end{aligned}
$$

where $J_{\mu}$ is the current operator, $f(x)$ is the Fermi distribution function, and we take the natural unit, i.e. $\hbar=c=1$. This is the general expression, and KL result can be obtained by expanding the above Kubo formula to the first order in $\lambda$. On the other hand, Smit [3] stressed the essential relevance of the impurity scattering, which disturbs the translational symmetry. Luttinger [4] reconsidered this problem by taking into account the impurity scattering in a systematic way, and essentially found that $\sigma_{x y}=\sigma_{K L}+\sigma_{L}$ where $\sigma_{K L}$ is the original result of KL [2] while the second term $\sigma_{L}$ is the new term which is proportional to the inverse of the impurity scattering potential. $\sigma_{L}$ is strongly dependent on the explicit form of the impurity scattering, and vanishes for the short range potential [5]. However most of the later theories regards the modification of the scattering events due to the spin-orbit interaction as the origin of AHE [6].

Very recently, it has been experimentally recognized that there are two distinct types of behaviors of AHE [8, 9, 10, 11, 12, 13]. In the conventional 
one, $\sigma_{x y}$ decreases as the temperature $T$ is lowered, and the AHE is attributed to the scattering events influenced by the spin-orbit interaction (type I). In the other case, $\sigma_{x y}$ increases as $T$ is lowered and remains finite in the limit $T=0$ (type II). In the type II systems, various anomalous behaviors of AHE are observed. For example, $\rho_{H}$ in thin films of $\mathrm{SrRuO}_{3}$ shows the change of magnitude of $10^{-9} \Omega m$ with the sign inversion $\| 10$ as a function of the temperature $T$ below the ferromagnetic transition temperature $T_{c}$. The change of $\sigma_{x y}$ per layer is estimated as $O\left(e^{2} / h\right)$ by using the data of the longitudinal resistivity $\rho \sim 10^{-7} \Omega m$ and the averaged lattice parameter $a \sim$ $4 \times 10^{-10} \mathrm{~m}$. (We appropriately recover Planck constant $h$.) Another example is the pyrochlore ferromagnet $\left(\mathrm{Sm}_{1-x} \mathrm{Ca}_{x}\right)_{2} \mathrm{Mo}_{2} \mathrm{O}_{7}$ showing the strong doping dependence of AHE, where hole doping of the order of $x=0.1$ causes the factor of $\sim 8$ change of $\rho_{H}$ in contrast to the almost unchanged $\rho_{x x}$ [12]. Similar phenomenon is observed also for the temperature dependence of $\rho_{H}$ in below $T=1 \mathrm{~K}\left(\ll T_{c} \cong 100 \mathrm{~K}\right)$ in $\mathrm{Nd}_{2} \mathrm{Mo}_{2} \mathrm{O}_{7}$ [13]. This huge sensitivity on $E_{F}$ and/or $T$ can not be understood in terms of the conventional theories on the AHE nor the change of $R_{0}$ below $T_{c}$. The purpose of this paper is to give a theoretical explanation for the distinction of type I and type II systems and the anomalous behaviors of $\sigma_{x y}$ in type II systems.

On the other hand, the topological nature of $\sigma_{x y}$ in 2D has been extensively studied in the context of quantum Hall effect under external magnetic field [14]. The Kubo formula eq.(2) for $\omega=0$ can be rewritten as 14

$$
\begin{aligned}
\sigma_{x y}(0)= & i \sum_{n, \vec{k}} f\left(\varepsilon_{n}(\vec{k})\right) \\
& \quad \times \sum_{m \neq n} \frac{\left\langle n \vec{k}\left|J_{y}\right| m \vec{k}\right\rangle\left\langle m \vec{k}\left|J_{x}\right| n \vec{k}\right\rangle-\left(J_{x} \leftrightarrow J_{y}\right)}{\left[\varepsilon_{n}(\vec{k})-\varepsilon_{m}(\vec{k})\right]^{2}} \\
= & e^{2} \sum_{n, \vec{k}} f\left(\varepsilon_{n}(\vec{k})\right)\left[\nabla_{k} \times \vec{A}_{n}(\vec{k})\right]_{z}
\end{aligned}
$$

where $\vec{A}_{n}(\vec{k})=-i\left\langle n \vec{k}\left|\nabla_{k}\right| n \vec{k}\right\rangle$. When the Fermi energy is in a gap and $T=0 \mathrm{~K}$, this integral is transformed to the "vorticity" corresponding to the vector potential $\overrightarrow{A_{n}}(\vec{k})$, and is an integer called Chern number [times $(2 \pi)^{-1}$ ].

From the viewpoint of the field theory, the appearance of $\sigma_{x y}$ in 2D is closely related to the parity anomaly in $(2+1) \mathrm{D}$ Dirac fermions, where the Chern-Simons term is generated with the sign depending on that of the 
fermion mass [15]. In the Bloch wave case, this Dirac fermion corresponds to the band crossing [16], and several models have been proposed for the condensed matter realization of the parity anomaly [17, 18]. Haldane [18] recognized that the quantum Hall effect can be realized as the result of the magnetic ordering and without external magnetic field in 2D. It should be noted here that parity transformation in $(2+1) \mathrm{D}$ is $\vec{r}=(x, y) \rightarrow \vec{r}^{\prime}=(-x, y)$, and this symmetry operation changes the sign of the Chern-Simons term. Therefore the effective Hamiltonian should break both $\mathrm{P}_{(2+1) \mathrm{D}^{-}}$and T-symmetries (at least infinitesimally).

Although the physical realization of Haldane's model [18], i.e., the complex transfer integrals between next-nearest-neighbor sites on the honeycomb lattice, is not so easy, more realistic model has been proposed in connection to the the spin chirality mechanism of AHE [19]. The spin chirality $\vec{S}_{i} \cdot \vec{S}_{j} \times \vec{S}_{k}$ is basically the solid angle subtended by the non-coplanar spin configurations, and its relevance to the AHE has been investigated in manganites at finite temperature [8] and in pyrochlore compounds [9, 11] at zero or low temperatures. This spin chirality acts as the effective magnetic field [20], and it has been found that the geometry of the lattice is crucial for the finite $\sigma_{x y}$ in terms of this mechanism [19]. Roughly speaking, the positive and negative fluxes in the unit cell should be inequivalent to avoid the cancellation. The multiband effect due to the existence of plural number of atoms in a unit cell is essential, and each band is characterized by the Chern number [14]. Therefore the two conditions, i.e., non-coplanar spins and non-trivial geometrical structure, makes this mechanism rather special.

In this letter we explore the connection between the AHE and the parity anomaly in 2D ferromagnets, and found the followings. (i) The parity anomaly and the resultant nonzero $\sigma_{x y}$ is not the special case but is the generic phenomenon occurring in $\mathrm{P}_{(2+1) \mathrm{D}^{-}}$and T-broken magnetic ordered state. Our model is the conventional one with the spin-orbit interaction $\lambda$ and the collinear spins on the simple square lattice (perovskite structure). (ii) The band crossings occur very often as we change the spin-orbit coupling $\lambda$ and/or the magnetization $m_{z}$. Even though the Fermi energy is in general not in a gap, these band crossings (Dirac fermions) behave as the topological singularities and appear as the sharp peaks and/or the sign change of $\sigma_{x y}$ as a function of the Fermi energy or the magnetization $m_{z}$.

We consider a model with triply degenerate $t_{2 g}$ orbitals with spin-orbit 
interaction on the $2 \mathrm{D}$ square lattice.

$$
\begin{aligned}
& H=-\sum_{i, \sigma} t_{0}\left[c_{x y \sigma}^{\dagger}(i) c_{x y \sigma}(i+x)+c_{x y \sigma}^{\dagger}(i) c_{x y \sigma}(i+y)\right. \\
& \left.\quad+c_{y z \sigma}^{\dagger}(i) c_{y z \sigma}(i+y)+c_{z x \sigma}^{\dagger}(i) c_{z x \sigma}(i+x)+\text { h.c. }\right] \\
& +\sum_{i, \sigma, \pm} \pm t_{1}\left[c_{x y \sigma}^{\dagger}(i) c_{z x \sigma}(i \pm y)+c_{x y \sigma}^{\dagger}(i) c_{y z \sigma}(i \pm x)+\text { h.c. }\right] \\
& +H_{S O}+H_{\mathrm{el}-\mathrm{el}}
\end{aligned}
$$

where the transfer integrals are determined by the oxygen $p$-orbitals between the two transition metal ions. $t_{0}$ is nonzero even for the perfect perovskite structure, while $t_{1}$ becomes nonzero due to the shift of the oxygen atoms out of plane in the positive (negative) $z$-direction. This will cause the Dzyaloshinsky-Moriya interaction [21] when the spin-orbit interaction $H_{S O}$ and the transfer integrals are combined, because the space inversion symmetry at the middle of the bond is broken. Our definition of $\mathrm{P}_{(2+1) \mathrm{D}}$ here is for the effective 2D Hamiltonian, and does not include the transformation on the orbital and spin degrees of freedom. The $t_{1}$-term breaks

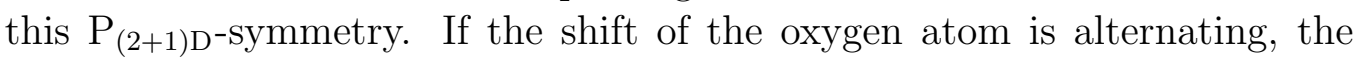

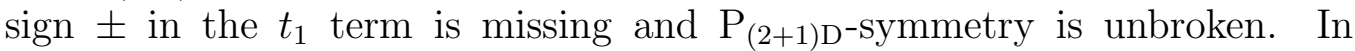
this case, the topological effect described below is missing, because the gauge flux density $b_{n}(\vec{k})=\left[\nabla_{k} \times \vec{A}_{n}(\vec{k})\right]_{z}$ in eq.(3) has the symmetry as $b_{n}\left(-k_{x}, k_{y}\right)=-b_{n}\left(k_{x}, k_{y}\right)$. (Note that $b_{n}(\vec{k})$ itself is not zero.) We approximate $H_{S O}$ by taking the matrix elements of the angular momentum $\vec{\ell}$ in the space of $t_{2 g}$ orbitals as

$$
H_{S O}=\lambda \sum_{i} \sum_{\alpha, \beta} \sum_{\sigma, \sigma^{\prime}} c_{\alpha \sigma}^{\dagger}(i) \vec{\ell}_{\alpha \beta} \cdot \vec{\tau}_{\sigma \sigma^{\prime}} c_{\beta \sigma^{\prime}}(i)
$$

where $\vec{\tau}$ are Pauli matrices and $\alpha, \beta=x y, y z, z x$. Although $H_{S O}$ is the on-site interaction, it can produce the effective gauge flux combined with the transfer integrals. The electron-electron interaction $H_{\mathrm{el}-\mathrm{el}}$ induces the ferromagnetic ordering and its details are not relevant to the present study. We take the mean field approximation as

$$
H_{\mathrm{el}-\mathrm{el}}=-\frac{U}{2} m_{z} \sum_{i, \alpha, \sigma} \sigma c_{\alpha \sigma}^{\dagger}(i) c_{\alpha \sigma}(i)
$$


where the ferromagnetic moment is along the $z$-direction. Now the $6 \times 6$ Hamiltonian matrix for each $\vec{k}$-point can be easily diagonalized, and 6 bands are obtains. Figure 1 shows the energy dispersion obtained for a set of parameters given in the caption. The near-degeneracies of the two bands are found at $\vec{k}=[0,0]$ and $[ \pm \pi / 2, \pm \pi / 2]$.

Fig.2(a) shows the typical example of $\sigma_{x y}$ as a function of $E_{F}$ for the same set of parameters as in Fig.1, while Fig.2(b) shows the variation as a function of the magnetization $m_{z}$ with the fixed $E_{F}=0$. Both of them vary rather rapidly with the magnitude of $e^{2} / h$ and have sharp peaks. To analyze this behavior, we first define the Chern number $C h_{n}$ for each band $n$ as $C h_{n}=\sum_{\vec{k} \in 1 \mathrm{stBZ}} 2 \pi\left[\nabla_{k} \times \vec{A}_{n}(\vec{k})\right]_{z}$. For the parameter set of Fig.1, the $C h_{n}$ 's are $(-1,-2,3,-4,5,-1)$ from the lower to higher energy band. Namely the spin-orbit interaction gives the non-trivial topological structure to the Bloch bands in the ferromagnets as in the case of quantum Hall system.

The next question is how the topological number appears in $\sigma_{x y}$. In the absence of the gap, one has to consider the distribution of $\left[\nabla_{k} \times \vec{A}_{n}(\vec{k})\right]_{z}$ in the $\vec{k}$-space. In Fig. 3 shown $\left[\nabla_{k} \times \vec{A}_{n}(\vec{k})\right]_{z}$ again for the parameter set in Figs.11 and 2(a), where sharp peaks near $\vec{k}=[0,0]$ and $\vec{k}=[ \pm \pi / 2, \pm \pi / 2]$ are clearly seen. This behavior can be understood as follows. When the band degeneracy occurs at $\vec{k}=\vec{k}_{0}$ for $u=U m_{z}=u_{c}$ or $\lambda=\lambda_{c}$, the electrons near this point can be described by the generalized Dirac fermions as

$$
\begin{aligned}
& H \cong \int \frac{d^{2} k}{(2 \pi)^{2}} \psi^{\dagger}(\vec{k}) \mathcal{H}(\vec{k}) \psi(\vec{k}), \quad \vec{\kappa}=\frac{1}{k_{D}}\left(\vec{k}-\vec{k}_{0}\right), \\
& \mathcal{H}(\vec{k})=\left[\begin{array}{cc}
V(\vec{k})+m v^{2} & v k_{D}\left(\kappa_{x}-i \kappa_{y}\right)^{p} \\
v k_{D}\left(\kappa_{x}+i \kappa_{y}\right)^{p} & V(\vec{k})-m v^{2}
\end{array}\right],
\end{aligned}
$$

where $\psi(\vec{k})$ is the two component spinor corresponding to the two crossing or touching bands, $v$ and $k_{D}$ are positive constants, and $V(\vec{k})$ is a function of $\vec{k}$. The usual Dirac fermion corresponds to the case $p=1$. By diagonalizing $\mathcal{H}(\vec{k})$, we get the dispersion around $\vec{k}_{0}$ as $\varepsilon_{ \pm}(\vec{k})= \pm \sqrt{\left(v k_{D} \kappa^{p}\right)^{2}+\left(m v^{2}\right)^{2}}+$ $V(\vec{k})$, where the sign $+/-$ represents the upper/lower band. The current operator is given by $\vec{J}=\int d^{2} k /(2 \pi)^{2} \psi^{\dagger}(\vec{k}) \nabla_{k} \mathcal{H}(\vec{k}) \psi(\vec{k})$. The mass $m$ is a continuous function of $(\lambda, u)$, and can changes sign across the critical lines in $(\lambda, u)$-plane. There the transfer of $C h_{n}$ occurs between these two bands[22]. Near this criticality, i.e., when the mass $|m|$ is small, the gauge flux density 
$b_{ \pm}(\vec{k})=\left[\nabla_{k} \times \vec{A}_{ \pm}(\vec{k})\right]_{z}$ is peaked near the degeneracy point as

$$
\begin{aligned}
b_{ \pm}(\vec{k}) & \cong i \frac{\left\langle \pm, \vec{k}\left|J_{y}\right| \mp, \vec{k}\right\rangle\left\langle\mp, \vec{k}\left|J_{x}\right| \pm, \vec{k}\right\rangle-\left(J_{x} \leftrightarrow J_{y}\right)}{\left[\varepsilon_{ \pm}(\vec{k})-\varepsilon_{\mp}(\vec{k})\right]^{2}} \\
& = \pm \frac{\left(p v \kappa^{p-1}\right)^{2} m v^{2}}{2\left[\left(v k_{D} \kappa^{p}\right)^{2}+\left(m v^{2}\right)^{2}\right]^{\frac{3}{2}}}
\end{aligned}
$$

The flux density $b_{ \pm}(\vec{k})$ contributes $\pm \frac{p}{2} \operatorname{sgn}(m)$ to the $C h_{n}$ of each band.

The transfer of $C h_{n}$ has been studied in connection with the usual Dirac fermion, i.e., $p=1$, mainly in the context of the integer quantum Hall effect 23]. However, in the present model, we have actually found also its generalizations given by eq.(7). For example, the type of $p=2$ is seen with the parameter set $\left(t_{1}=0.5 t_{0}, \lambda \sim 0, U m_{z}=5 t_{0}\right)$, and the transfer of $C h_{n}$ at each degeneracy point is \pm 2 when $\lambda$ is changed across zero. Each band touches the next band with the energy dispersion $E(\vec{k}) \propto \vec{k}^{2}$. Another remark here is that the band crossing and the transfer of $C h_{n}$ occurs frequently. It occurs at least 6 times for the parameter set $\left(t_{1}=0.5 t_{0}, \lambda=0.4 t_{0}\right)$ when $U m_{z}$ is changed from 0 to $5 t_{0}$. It occurs more often if we additionally change $\lambda$.

Some remarks are now in order. One is on the effect of disorder, which has been neglected thus far. It is well known that the extended states in 2D is unstable against the infinitesimal disorder in the absence of magnetic field. On the other hand, the topological $C h_{n}$ should be quantized and stable as far as the impurity scattering strength is weak enough compared with the gap of the Dirac fermions. This is the basis of the existence of the extended states and plateau of $\sigma_{x y}$ in the integer QHE, and should work here also [24]. Therefore we expect that the random potential realizes the quantum Hall effect in 2D ferromagnets.

Second remark is on the three dimensional case. The crossing of bands also plays an important role here, but the singularity is more mild. According to the analysis of Dirac fermions in 3D, the peak of $\sigma_{x y}$ near the critical point is of the order of $|m|$ and the width is again $|m|$. The magnitude of $\sigma_{x y}$ is of the order of $|m| / t_{0}$ in this case, but it varies over the energy scale of $t_{0}$ except near the singularity. Near the singularity, $\sigma_{x y}$ changes rapidly with the energy scale of $|m|$ while the magnitude remains of the order of $|m| / t_{0}$.

Now the experimental relevance of the present results is briefly discussed. The most significant consequence of the topological nature of AHE is its 
sensitivity to the Fermi energy $E_{F}$ and/or the magnetization, in contrast to the diagonal conductivity $\sigma_{x x}$ [25]. This naturally explains the anomalous features of AHE in thin films of $\mathrm{SrRuO}_{3}$ [10] and the single crystal of pyrochlore Mo oxides [12, 13] mentioned on the introduction. Especially it has been known that the topological mechanism for AHE is relevant in the latter material [11]. The 2D system is even more interesting, where the variation in $\sigma_{x y}$ of the order of $e^{2} / h$, and with the impurity scattering the quantum Hall effect can be also expected. It is noted here that the conventional formula eq.(11) does not work in this case, and $\sigma_{x y}$ should be plotted instead. In conclusions, we have studied the anomalous Hall effect from the viewpoint of the topology and parity/chiral anomaly. When both the parity and time-reversal symmetries are broken, the AHE survives even at $T=0$ where the periodicity is recovered ( type II). In this case, the band crossing behaves as the topological singularity causing the anomalous behavior of $\sigma_{x y}$ as a function of the temperature $T$, the magnetization $m_{z}$, and/or the Fermi energy $E_{F}$.

The authors acknowledges Y. Tokura, H. Fukuyama, and Y. Taguchi, for fruitful discussions. N. N. is supported by Priority Areas Grants and Grantin-Aid for COE research from the Ministry of Education, Science, Culture and Sports of Japan,

\section{References}

[1] A. W. Smith and R. W. Sears, Phys. Rev. 34 (1929) 1466.

[2] R. Karplus and J. M. Luttinger, Phys. Rev. 95 (1954) 1154.

[3] J. Smit, Physica 24 (1958) 39.

[4] J. M. Luttinger, Phys. Rev. 112 (1958) 739.

[5] H. Fukuyama, Ph. D thesis (1970) unpublished.

[6] J. Kondo, Prog. Theor. Phys. 27 (1962) 772; P. Nozieres and C. Lewiner, J. Phys. (Paris) 34 (1973) 901.

[7] R. Kubo, J. Phys. Soc. Jpn. 12 (1957) 570. 
[8] P. Matl et al., Phys. Rev. B 57 (1998) 10248; J. Ye et al., Phys. Rev. Lett. 83 (1999) 3737; Y. Taguchi and Y. Tokura, Phys. Rev. B 60 (1999) 10280; S. H. Chun et al., Phys. Rev. Lett. 84 (2000) 757.

[9] T. Katsufuji, H. Y. Hwang, and S-W. Cheong, Phys. Rev. Lett. 84 (2000) 1998; S. Yoshii et al., J. Phys. Soc. Jpn. 69 (2000) 3777.

[10] M. Izumi et al., J. Phys. Soc. Jpn. 66 (1997) 3893; L. Klein et al., Phys. Rev. B 61 (2000) R7842.

[11] Y. Taguchi et al., Science 291 (2001) 2573.

[12] Y. Taguchi and Y. Tokura, Europhys. Lett. 54 (2001) 401.

[13] M. Sato, private communications.

[14] D. J. Thouless et al., Phys. Rev. Lett. 49, 405 (1982); M. Kohmoto, Ann. Phys. (N.Y.) 160 (1985) 343.

[15] S. Deser, R. Jackiw, and S. Templeton, Phys. Rev. Lett. 48 (1982) 975; R. Jackiw, Phys. Rev. D 29 (1984) 2375.

[16] H. B. Nielson and M. Ninomiya, Phys. Lett. 130B (1983) 389.

[17] G. W. Semenoff, Phys. Rev. Lett. 53 (1984) 2449; E. Fradkin, E. Dagotto, and D. Boyanovsky, Phys. Rev. Lett. 57 (1986) 2967.

[18] F. D. M. Haldane, Phys. Rev. Lett. 61 (1988) 2015.

[19] K. Ohgushi, S. Murakami, and N. Nagaosa, Phys. Rev. B 62 (2000) R6065.

[20] P. W. Anderson and H. Hasegawa, Phys. Rev. 100 (1955) 675; X. G. Wen, F. Wilczek and A. Zee, Phys. Rev. B 39 (1989) 11413; P. A. Lee and N. Nagaosa, Phys. Rev. B 46 (1992) 5621.

[21] I. Dzyaloshinsky, J. Phys. Chem. Solids 4 (1958) 241; T. Moriya, Phys. Rev. 120 (1960) 91.

[22] J. E. Avron, R. Seiler, and B. Simon, Phys. Rev. Lett. 51 (1983) 51; B. Simon, Phys. Rev. Lett. 51 (1983) 2167. 
[23] Y. Hatsugai and M. Kohmoto, Phys. Rev. B 42 (1990) 8282; M. Oshikawa, Phys. Rev. B 50 (1994) 17357.

[24] S. Kivelson, D. H. Lee, and S. C. Zhang, Phys. Rev. B 46 (1992) 2223; D. N. Sheng and Z. Y. Weng, Phys. Rev. Lett. 78 (1997) 318.

[25] The magnetization $m_{z}$ changes smoothly as $T$ is lowered below $T_{c}$ and $\sigma_{x y}$ changes as a function of $T$ tracing the $U m_{z}$ axis of Fig.2(b) We thank Y.Tokura for suggesting this possibility. 


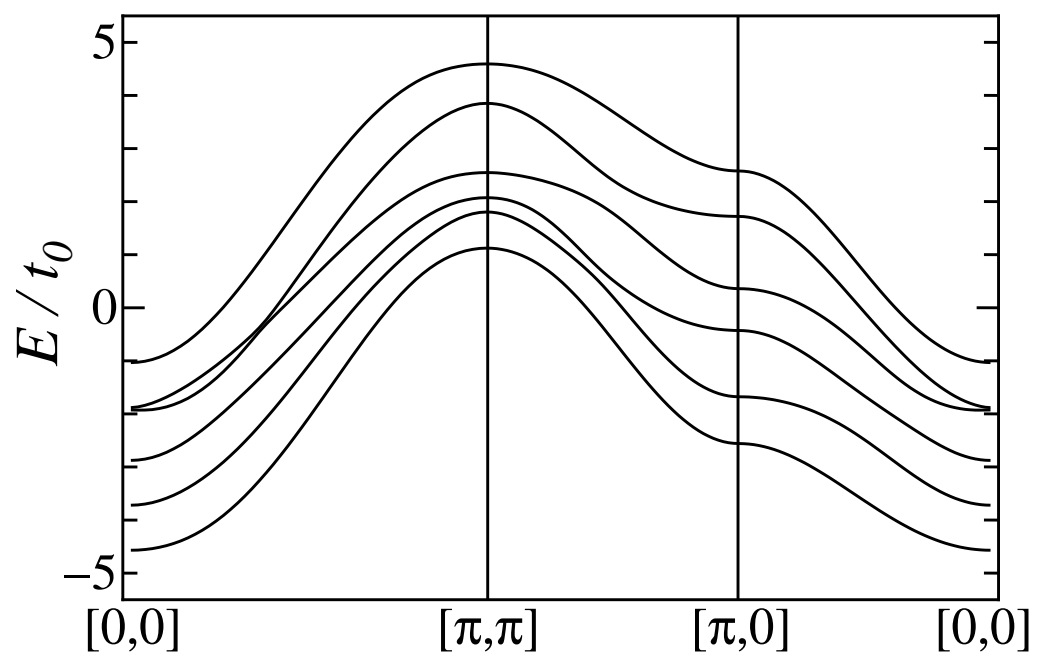

Figure 1: Dispersion of 6 bands for $t_{1}=0.5 t_{0}, \lambda=0.4 t_{0}, U m_{z}=0.95 t_{0}$. The 4 th and 5 th bands are nearly degenerate at $\vec{k}=[0,0]$ and $[ \pm \pi / 2, \pm \pi / 2]$. 

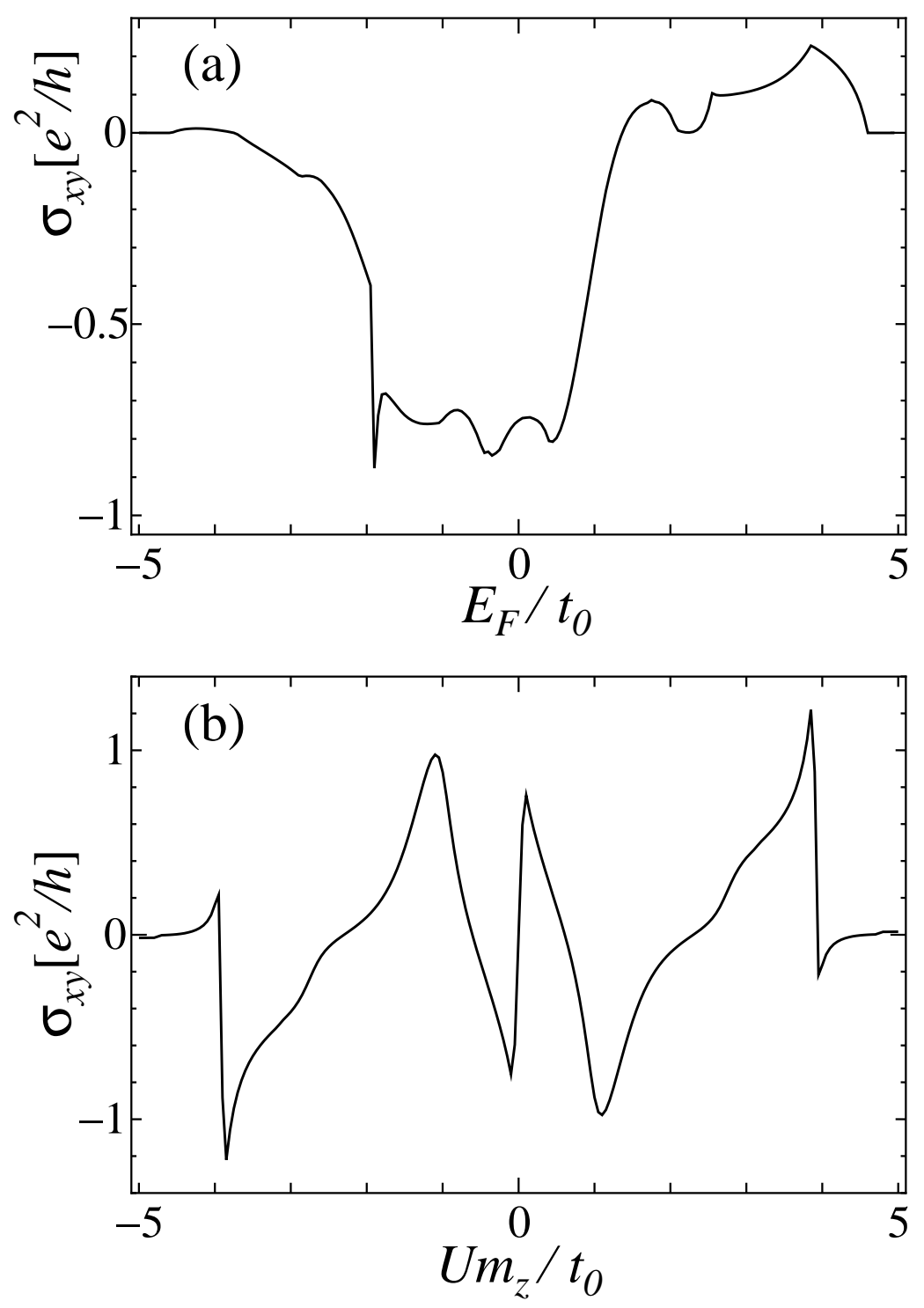

Figure 2: (a) Transverse conductivity $\sigma_{x y}$ as a function of the Fermi energy $E_{F}$ with the parameter set $t_{1}=0.5 t_{0}, \lambda=0.4 t_{0}, U m_{z}=0.95 t_{0}$. The sharp peak near $-2 t_{0}$ is due to the near-degeneracy point at $\vec{k}=[0,0]$ between the 4th and 5th bands (see Fig.(1), and, in other words, due to the sharp peak in the gauge flux density in $\vec{k}$-space (see Fig.33). (b) Transverse conductivity $\sigma_{x y}$ as a function of the magnetization $m_{z}$ [times $U$ ] with $t_{1}=0.5 t_{0}, \lambda=0.4 t_{0}$, $E_{F}=0$. 

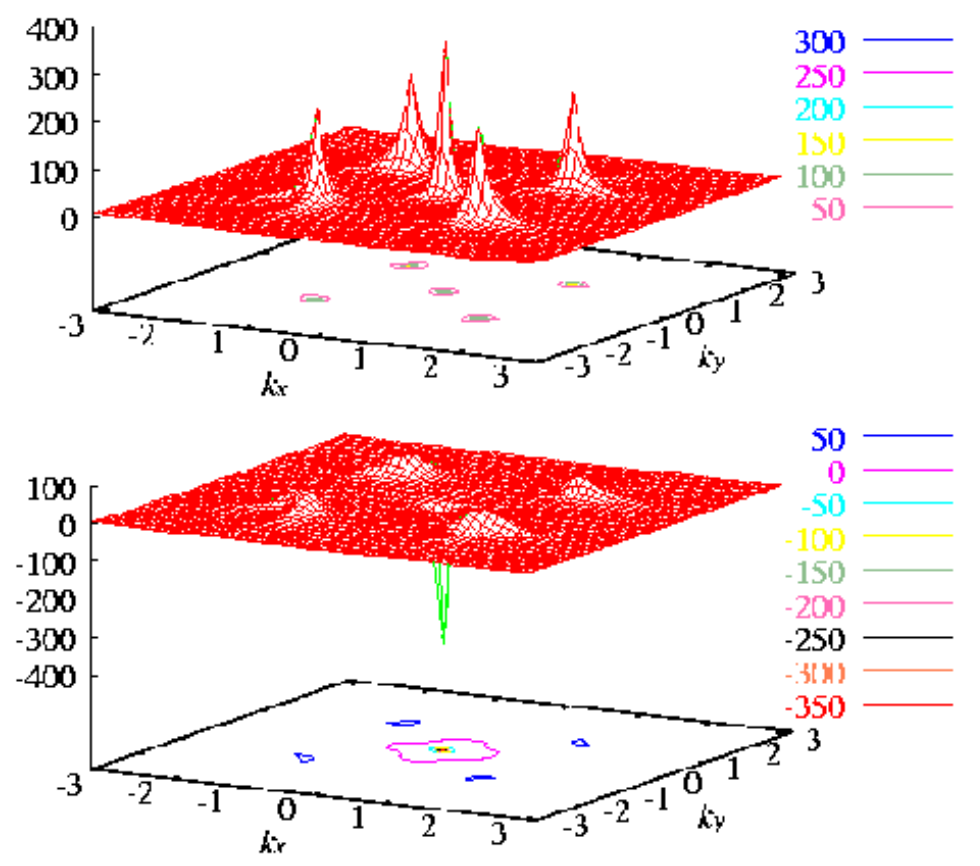

Figure 3: Gauge flux density in $\vec{k}$-space of the 5 th band with the parameter sets $t_{1}=0.5 t_{0}, \lambda=0.4 t_{0}, U m_{z}=0.95 t_{0}$ for the upper, and $U m_{z}=1.05 t_{0}$ for the lower. The transfer of $C h_{n}$ occurs between the 4 th and 5 th bands at $\left(U m_{z}\right)_{c} \sim 1.0 t_{0} .5$ peaks are found in both parameter sets. However, the transfer occurs only at $\vec{k}=[0,0]$ in this case. 